\title{
Kommunikationsprobleme zwischen autonomen Fahrzeugen und menschlichen Fahrern
}

\author{
Berthold Färber
}

\section{Inhaltsverzeichnis}

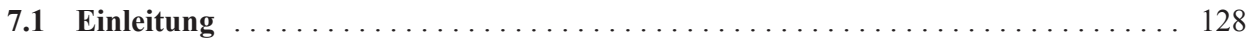

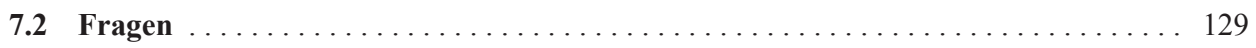

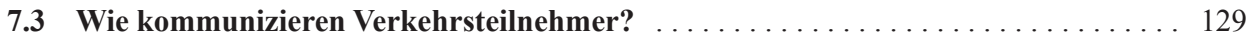

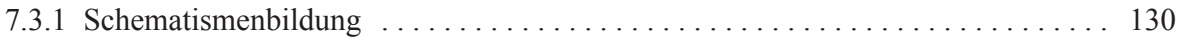

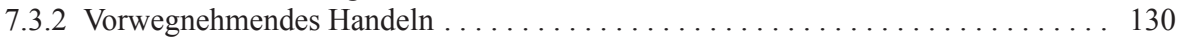

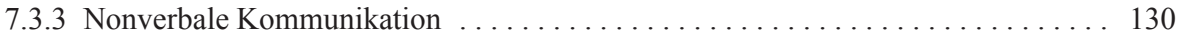

7.3.4 Gesichtsausdruck und Augenkontakt . . . . . . . . . . . . . . . . 131

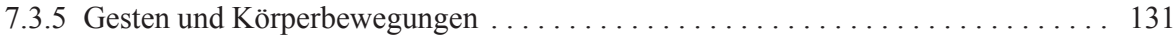

7.4 Auswirkungen der Kommunikationsmöglichkeiten

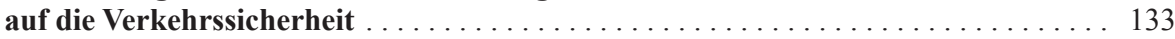

7.4.1 Sonderfälle: Polizisten und Fahrzeuge mit Sonderrechten erkennen . . . . . . . . . . 134

7.5 Auswirkungen der Kommunikationsmöglichkeit auf die Akzeptanz autonomer

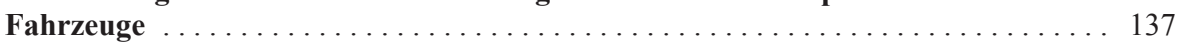

7.6 Mit welchem mentalen Modell werden andere Verkehrsteilnehmer auf Fahrfehler

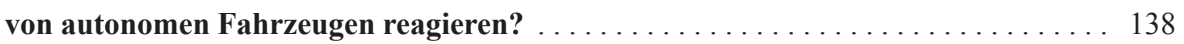

$7.7 \quad$ Kulturelle Unterschiede . . . . . . . . . . . . . . . . . . . . . . . . . . . . . . . 139

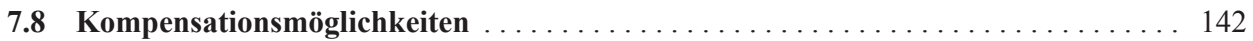

7.9 Neue Kommunikationsformen für einen effektiven Informationsaustausch

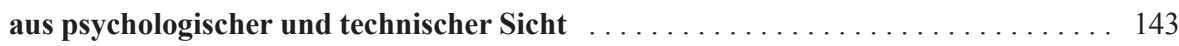

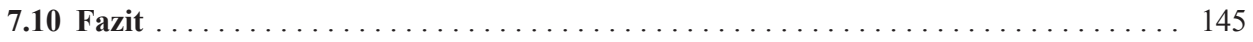

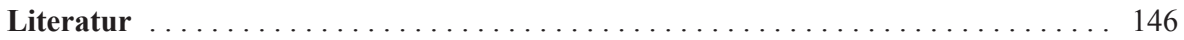

B. Färber $(\square)$

Universität der Bundeswehr München, Deutschland berthold.faerber@unibw.de 


\subsection{Einleitung}

Häufig wird im Zusammenhang mit autonomen Landfahrzeugen der Luftverkehr als Beispiel herangezogen, bei dem - abgesehen von Start und Landung - der Autopilot die Steuerung übernimmt. Somit stellt sich die Frage: Was können wir aus dem Luftverkehr lernen? Die Gemeinsamkeit zwischen autonom fliegenden und autonom fahrenden Fahrzeugen besteht darin, dass der Pilot bzw. Fahrer die letzte Verantwortung trägt. Es gibt aber mehrere Unterschiede zwischen Straßenverkehr und Luftverkehr (und damit ist nicht die Art der Fortbewegung gemeint), die eine Übertragung zwischen den Systemen nicht sinnvoll macht. Ein wesentlicher Unterschied, der im vorliegenden Beitrag von zentraler Bedeutung ist, ist die Anwendung von Regeln sowie die Art der Steuerung. In Bereichen, in denen Flugzeuge aufeinandertreffen - also vor allem beim Rollverkehr - ist alles durch strikte Regeln festgelegt. Weiterhin gibt es eine übergeordnete Überwachung und Steuerung, die dem Piloten exakte Anweisungen gibt und in Zweifelsfällen, die möglicherweise nicht durch Regeln gedeckt sind, Entscheidungen trifft und an den Piloten kommuniziert. Der Pilot hat also in diesen Situationen keinerlei Ermessens- oder Entscheidungsspielraum. Er ist ausschließlich ein ausführendes Organ. Im Vergleich zum Luftverkehr stellt der Straßenverkehr eher ein selbstorganisiertes, chaotisches System dar, das zwar prinzipiell durch Regeln geordnet wird, bei dem aber viele Situationen nicht in einer eindeutigen Regel festgelegt werden können. In diesem Fall kommt dann immer $\S 1$ der Straßenverkehrsordnung (StVO) zur Anwendung, der besagt: „Die Teilnahme am Straßenverkehr erfordert ständige Vorsicht und gegenseitige Rücksicht“, und „Wer am Verkehr teilnimmt, hat sich so zu verhalten, dass kein anderer geschädigt, gefährdet oder mehr, als nach den Umständen unvermeidbar, behindert oder belästigt wird.“ Im Sinne eines erschöpfenden Regelwerks für das Verhalten in allen denkbaren Situationen gibt es also im Straßenverkehr eine große Kategorie „Sonstige“, die von Verkehrsteilnehmern im Einvernehmen und unter Berücksichtigung von $\S 1$ STVO gelöst werden müssen. Somit gehört zu den zentralen Aufgaben eines Fahrers zur sicheren Teilnahme am Straßenverkehr auch die Einschätzung des Verhaltens anderer Verkehrsteilnehmer. Diese Einschätzung und Vorhersage des Verhaltens anderer Verkehrsteilnehmer beruht zunächst auf der Annahme von mehr oder weniger regelkonformem Verhalten der anderen. Zu der Absichtsabschätzung gehört aber auch die Kommunikation der Fahrer und Verkehrsteilnehmer untereinander durch Aktionen und Zeichen. Mit anderen Worten: Neben den „offiziellen“ Regeln existiert ein Satz von informellen Regeln, die den Verkehr steuern.

Wer als Tourist in anderen Ländern unterwegs ist, sei es als Autofahrer oder Fußgänger, kann aus eigener Anschauung erleben, wie sehr informelle Regeln, kulturspezifische Verhaltensweisen und die Kommunikation zwischen Verkehrsteilnehmern den Verkehr bestimmen. Trifft man als menschlicher Fahrer auf diesen anders gearteten „Regelkanon“, so erlebt man zunächst eine Phase der Irritation. In der zweiten Phase der Adaptation passt man sich im Laufe der Zeit mehr unbewusst an diese neuen, nirgendwo exakt fixierten Regeln an. In der Phase der Konsolidierung erscheinen sie als selbstverständlich, obwohl sie nicht fixiert und teilweise nicht einmal exakt zu beschreiben sind. 
Der motorisierte Straßenverkehr stellt ein System mit sehr unterschiedlichen Akteuren dar, das zum Ziel hat, die Teilnehmer sicher, störungsfrei und schnell zum Ziel zu bringen. Unter Systemgesichtspunkten ist unmittelbar einleuchtend, dass die Beachtung informeller Regeln, die Kommunikation zwischen den Verkehrsteilnehmern und Verhaltensvorhersage anderer zwei wichtige Funktionen erfüllt: Sie unterstützen zum einen den Verkehrsfluss und dienen zum anderen zur Kompensation von Fehlern.

\subsection{Fragen}

In technischen Termini ausgedrückt sind Menschen multisensorische lernfähige Systeme. Das bedeutet, sie sind in der Lage, verschiedene, auch schwache und nicht eindeutige Signale zu einem Gesamtbild zu vereinen und zu interpretieren. Sie sind weiterhin in der Lage, sich verändernden Gegebenheiten anzupassen und so die beiden o. g. Funktionen Kompensation der Fehler anderer und Verbesserung des Verkehrsflusses - zu unterstützen. Was aber passiert, wenn nicht nur Menschen, sondern zusätzlich Roboter-Fahrzeuge am Verkehr teilnehmen, die sich streng an Regeln halten und die informellen Regeln sowie Teile der Kommunikation nicht verstehen? Wie reagieren menschliche Fahrer auf diese neuen Verkehrsteilnehmer, speziell in der Übergangsphase, in der die Fahrroboter noch die Minderheit stellen? Sind Menschen in der Lage, schwierige oder scheinbar unauflösbare Situationen kooperativ mit Fahrrobotern zu lösen? Schließlich, über welche Eigenschaften oder Kennzeichnungen müssten Fahrroboter minimal verfügen, um am gemischten Verkehr mit menschlichen Fahrern problemlos teilnehmen zu können?

Diesen Fragen will sich der Beitrag von verschiedenen Seiten nähern. Da autonome Fahrzeuge bislang hauptsächlich unter dem Aspekt technischer Performanz untersucht wurden wie bei der Urban challenge [1] oder der Berta-Benz-Fahrt [2] bzw. im Mischverkehr stets ein Sicherheitsfahrer an Bord war, um im Zweifelsfall einzugreifen (z. B. beim Stadtpilot [3]), existiert zu den oben angeführten Fragen kaum originäre Literatur. Vieles wird daher aus den Erkenntnissen anderer Wissenschaftsbereiche abzuleiten sein.

\subsection{Wie kommunizieren Verkehrsteilnehmer?}

Neben den vorgeschriebenen Zeichen zum Anzeigen einer Absicht wie Blinker, Warnblinkanlage, Bremslicht, Hupe und Lichthupe kommunizieren Verkehrsteilnehmer über eine Reihe ,informeller“ Kommunikationskanäle. Diese Kommunikation im Straßenverkehr ist - im Vergleich zur normalen menschlichen Kommunikation - durch eine eingeschränkte Verständigungsmöglichkeit geprägt. Nach Merten [4] gibt es verschiedene Möglichkeiten der Kommunikation. 


\subsubsection{Schematismenbildung}

Bei der Schematismenbildung wird aufgrund von bestimmten Eigenschaften eines Verkehrsteilnehmers auf dessen Verhalten geschlossen. Beispielsweise wird sich eine ältere Person mit Mobilitätsproblemen anders verhalten als ein Kind. Dem Fahrer eines Sportwagens unterstellt man ein anderes Fahrverhalten als dem Fahrer einer großen Limousine. Diese Schematismen treffen im Fahralltag natürlich nicht immer zu. Sie funktionieren allerdings im Prinzip und helfen, das Gesamtsystem Verkehr zu stabilisieren.

\subsubsection{Vorwegnehmendes Handeln}

Durch kleine Handlungsschritte ist die Richtung des eigenen Verhaltens für andere Verkehrsteilnehmer frühzeitig erkennbar. Beispiele: Nähert sich ein Fahrzeug dem linken Fahrstreifen (ohne den Blinker zu setzen), so deutet dies auf einen beabsichtigen Spurwechsel hin. Geht ein Fußgänger zielstrebig auf einen Zebrastreifen zu, so wird der Autofahrer annehmen, dass er die Straße an dieser Stelle überqueren will.

\subsubsection{Nonverbale Kommunikation}

Bei den informellen Kommunikationskanälen spielt die nonverbale Kommunikation vor allem in sogenannten Verhandlungssituationen eine Rolle. Nonverbale Kommunikation ist als Verständigungsform sicher die älteste Form der Verständigung zwischen Lebewesen. Wissenschaftlich hat sich schon Charles Darwin im Jahr 1874 in seinem Buch Der Ausdruck der Gemüthsbewegungen beim Menschen und den Thieren [5] mit nonverbaler Kommunikation beschäftigt. Wie subtil nonverbale Signale sind, ist aus einer alten Studie von Pfungst [6] über den ,klugen Hans“ bekannt, ein Pferd, das aufgrund unbewusster minimaler Signale seines Besitzers oder des Auditoriums „rechnen“ konnte. Die dafür wichtige Erkenntnis lautet, dass nonverbale Signale auch unbewusst ausgesendet werden und daher einer Analyse nicht immer leicht zugänglich sind.

Prinzipiell lassen sich nonverbale Signale in drei Bereiche einteilen:

- Gesichtsausdruck und Augenkontakt,

- Gesten und Körperbewegungen,

- Stimme und Art des Ausdrucks.

Für den Anwendungsfall „Straßenverkehr“ sind nur Gesichtsausdruck/Augenkontakt und Gesten/Körperbewegungen relevant und sollen daher weiter verfolgt werden. 


\subsubsection{Gesichtsausdruck und Augenkontakt}

Im Innerortsverkehr spielt der Blickkontakt zwischen Autofahrern, aber auch zwischen Autofahrern und anderen Verkehrsteilnehmern (Fußgängern, Radfahrern) eine wesentliche Rolle. Ein Fußgänger, der eine Straße ohne Querungshilfe überqueren will, versichert sich mittels Blickkontakt zum Autofahrer, dass er gesehen wird. Wird der Blick vom Autofahrer erwidert, so geht der Fußgänger davon aus, dass er gesehen wird und der Autofahrer Rücksicht nimmt [7].

Beim Einscheren aus einer untergeordneten Straße und dichtem Verkehr auf der bevorrechtigten Straße vergewissert sich der Einscherende ebenfalls mittels Blickkontakt, dass er - trotz sehr geringer Zeitlücke - einscheren darf, da der Fahrer auf der bevorrechtigten Straße seine Geschwindigkeit situationsangepasst reduziert.

Der Blickkontakt ist eine dyadische Kommunikation, d.h., der Blick wird von demjenigen, der angesehen wird, erwidert bzw. nicht erwidert. Wendet der Angesehene seinen Blick ab, so gibt er zu verstehen, dass er den anderen ,nicht gesehen“ hat, also auf sein Zeichen und die Verhandlung nicht eingehen will. Bezüglich der Auswirkungen dieser Strategie auf autonome Fahrzeuge ist zu unterscheiden zwischen autonomen Fahrzeugen, bei denen der Fahrersitz besetzt oder unbesetzt ist.

Ist der Sitz des Fahrers besetzt, so gibt es zwei Möglichkeiten:

3. Der „Fahrer“, d. h. die Person auf dem Fahrersitz, ist mit Tätigkeiten im Innenraum befasst und folglich kommt kein Blickkontakt zustande. In diesem Fall kann der andere Verkehrsteilnehmer nicht davon ausgehen, dass sein Verhandlungsangebot angenommen wird, und wird sich entsprechend verhalten.

4. Der Fahrer des autonomen Fahrzeugs betrachtet die Umgebung, weshalb sich die Blicke der beiden Verkehrsteilnehmer treffen, wenn auch eher zufällig. Hier kann der Blickkontakt bei dem nicht-autonomen Verkehrsteilnehmer zu einer falschen Einschätzung der Situation und damit zu einem Konflikt führen.

Ist der Fahrersitz nicht besetzt so erhält der andere Verkehrsteilnehmer keine Information, d. h., die Situation ist wie bei dem Szenario „,kein Blickkontakt“.

\subsubsection{Gesten und Körperbewegungen}

Gesten sind eine weit verbreitete und effektive Art der Verständigung zwischen Verkehrsteilnehmern. Viele Zeichen, die durch Gesten dargestellt werden, sind auch allgemein verständlich und weitgehend eindeutig. So drückt Nicken das Einverständnis mit dem Ansinnen oder der Bitte des anderen aus. Bewegt ein Fußgänger die Arme seitwärts auf und ab, so fordert er Fahrzeuge zum Anhalten auf, beispielsweise um eine Unfallstelle abzusichern. Die Handbewegung von oben nach unten (s. Abb. 7.1) wird eingesetzt, um anderen zu sagen, dass sie langsamer fahren sollen. Eine wischende Handbewegung (s. Abb. 7.2) 
Abb. 7.1 Slow-down (Quelle: [8])

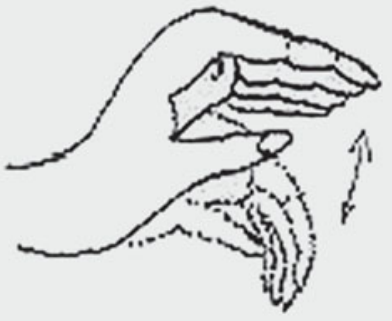

Abb. 7.2 Wischende Handbewegung (Quelle: [8])

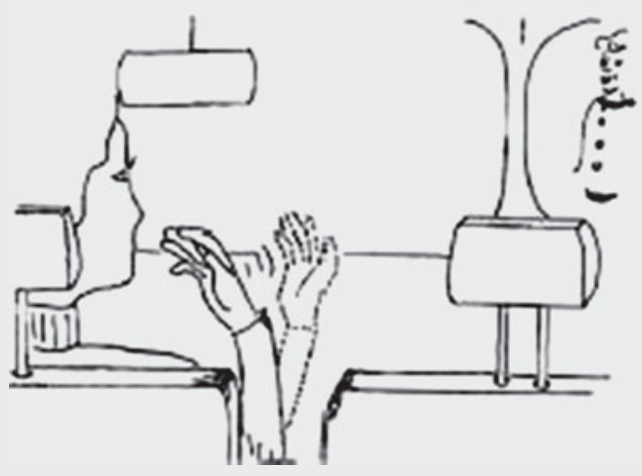

Abb. 7.3 Anbietende Geste 
oder die anbietende Geste mit nach oben zeigender Handfläche als Geste (s. Abb. 7.3) bedeutet: Bitte fahre oder gehe Du zuerst - ich verzichte auf mein Vorrecht.

\subsection{Auswirkungen der Kommunikationsmöglichkeiten auf die Verkehrssicherheit}

Auf den ersten Blick scheint es, als wären die informellen Zeichen wie Blickkontakt, Gesten oder vorwegnehmendes Handeln weniger eindeutig als die vorgeschriebenen Zeichen wie Blinker, Hupe, Lichthupe etc. Dies ist aber keineswegs der Fall. Es ist interessant, dass der Philosoph Heidegger, der sich in seiner Monografie Sein und Zeit [9] mit der Eigenschaft von Zeichen auseinandersetzt, das Auto als Beispiel wählt: „An den Kraftwagen ist neuerdings ein roter, drehbarer Pfeil angebracht, dessen Stellung jeweils, zum Beispiel an einer Wegkreuzung, zeigt, welchen Weg der Wagen nehmen wird“" (zitiert nach Frerichs, [10], S. 138). Vielleicht war damals dieses Zeichen noch ein-eindeutig und unabhängig von der Situation, jedoch wird schnell deutlich, dass Signale in unterschiedlichen Kontexten unterschiedliche Bedeutung besitzen. Alle Zeichen, d.h. verbale und nonverbale, sind nur in der Verknüpfung und im Kontext verständlich. Wie Savigny [11] ausführt, muss zwischen Signal und Äußerungsbedeutung unterschieden werden. Eine Lichthupe hat je nach Situation eine unterschiedliche Äußerungsbedeutung. Die Betätigung der Lichthupe durch einen Fahrer, der Vorfahrt hat und verzögert, wird als Angebot zum Einscheren verstanden, beschleunigt er oder fährt mit gleicher Geschwindigkeit weiter, so drückt das gleiche Signal aus, dass er auf seiner Vorfahrt beharrt. Auch der rechts gesetzte Blinker kann bedeuten: „Ich biege ab.“ oder „Ich fahre langsamer, weil ich eine Parklücke suche, und du kannst oder sollst überholen."

Mit Blick auf die schwächeren Verkehrsteilnehmer ist noch das Beispiel „Fahrradfahrer“ interessant. Radfahrer verwenden noch die Zeichen aus den Anfängen der Motorisierung zur Anzeige einer beabsichtigten Richtungsänderung. Das bedeutet für die Mustererkennung, dass - abgesehen vom Verkehr auf Autobahnen - nicht nur der Fahrradfahrer an sich, sondern auch seine Handzeichen sicher erkannt werden müssen.

Was bedeutet das für die Verkehrssicherheit? Zunächst ist davon auszugehen, dass autonome Fahrzeuge die mehrfache und kontextabhängige Bedeutung der Signale nicht kennen und sich daher sehr konservativ verhalten müssen. So lange eine sichere Blickerfassung oder das Handzeichen eines Fußgängers nicht sicher erfassbar sind und unter Berücksichtigung der Situation interpretiert werden können, so lange müssen Fußgänger, die sich auf einem potenziellem Kollisionskurs befinden, als Gefahr interpretiert werden und eine entsprechende Reaktion des autonomen Fahrzeugs auslösen. 


\subsubsection{Sonderfälle: Polizisten und Fahrzeuge mit Sonderrechten erkennen}

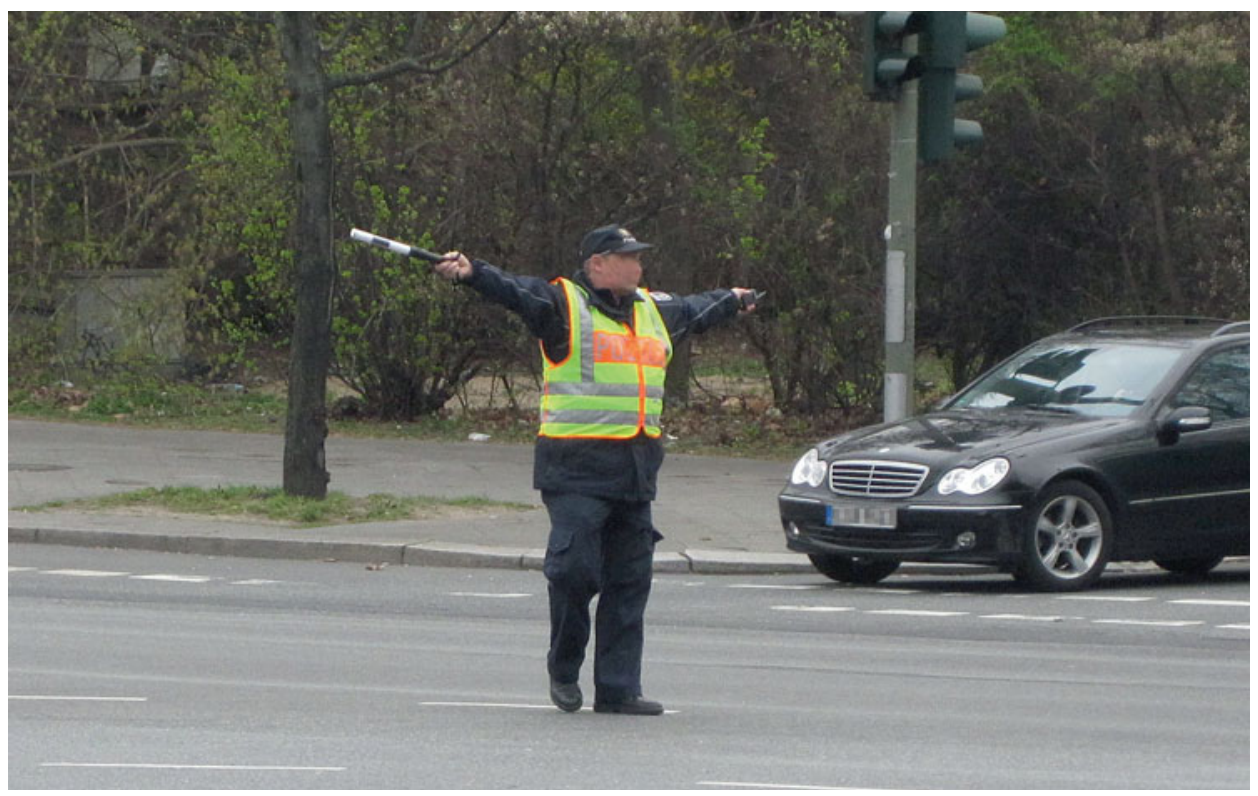

Abb. 7.4 Verkehrsposten: Verkehr fließt

Abb. 7.5 Verkehrsposten (Nord-Korea): Brust dem Fahrer zugewandt bedeutet „Stop“

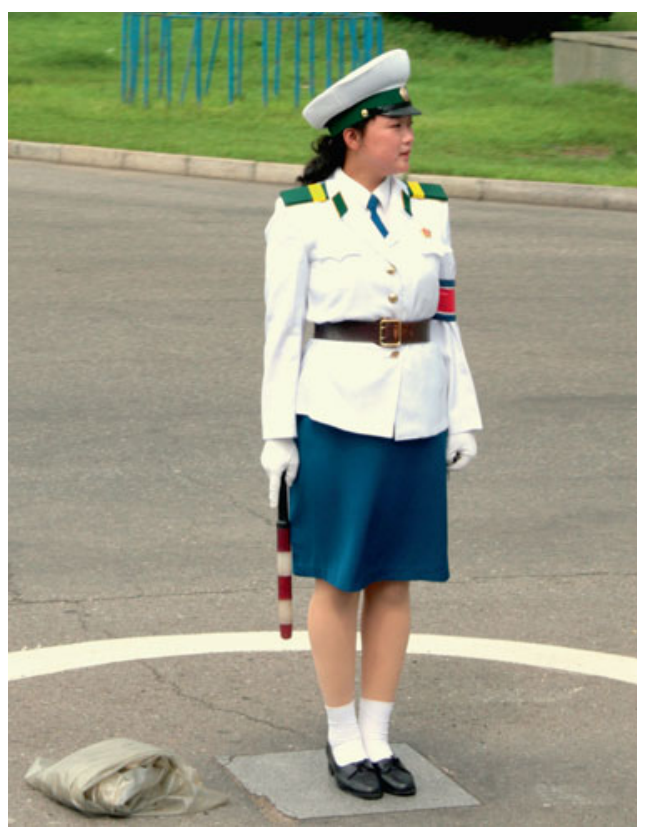


Erkennen autonome Fahrzeuge Fußgänger auf der Fahrbahn, so werden sie anhalten. Was passiert aber, wenn der Fußgänger ein Polizist ist, der den Verkehr regelt? Zum einen muss der Polizist als stehendes Hindernis erkannt werden, dem der Fahrroboter ausweichen muss. Darüber hinaus gilt es aber, die Zeichen des Polizisten richtig zu interpretieren. Verhält sich dieser streng regelkonform, wie in den Abb. 7.4 und Abb. 7.5 gezeigt, so kann ein autonomes Fahrzeug die entsprechende Bedeutung lernen. Aus Erfahrung ist aber bekannt, dass Polizisten, die den Verkehr regeln oder Einweiser auf Parkplätzen auch sehr dynamische Gesten verwenden, um den Verkehr zu beschleunigen, also mit den Armen oder Händen winken oder mit den Armen rudern (s. Abb. 7.6, Abb. 7.7 und Abb. 7.8). Menschen verstehen diese Zeichen vor allem aus dem Kontext heraus. Autonome Fahrzeuge müssten - neben der Fähigkeit zur Mustererkennung für Gesten und Körperhaltungen, auch über ein Kontextwissen verfügen, das ihnen die richtige Erkennung und Bewertung der Gesten ermöglicht.

Einen Spezialfall stellt die Berücksichtigung von Fahrzeugen mit Sonderrechten, also Einsatzfahrzeugen von Polizei, Feuerwehr oder Rettungsfahrzeugen dar. Diese Fahrzeuge machen zunächst mit akustischen Signalen auf sich aufmerksam. Optische und akustische Warnsysteme (in Deutschland Martinshorn und Blaulicht) verpflichten den übrigen Verkehr zum Anhalten, zur Gewährung der Vorfahrt an Kreuzungen oder zum Öffnen einer Rettungsgasse. Im täglichen Straßenverkehr werden Fahrzeuge noch nicht anhalten, wenn von Ferne das akustische Signal zu hören ist. Ein derartiges Verhalten würde den Verkehr zu sehr beeinträchtigen, z.B. in der Nähe eines Krankenhauses. Zudem ist eine exakte Lokalisierung der Richtung, aus der das akustische Signal kommt, bei größerer Entfernung nicht möglich. Auch die Erkennung des Sonderfahrzeugs in größerer Entfernung und die fahrspurbezogene Zuordnung dürften sich ebenfalls schwierig gestalten. Deshalb müssten autonome Fahrzeuge aus Sicherheitsgründen bei jedem dieser Signale anhalten, um den Verkehr nicht zu gefährden. Wie sich das auf die Akzeptanz auswirkt, wird in der Folge näher analysiert.

Abb. 7.6 Ein Polizist in Minneapolis gibt Zeichen, dass der Verkehr auf einer Straßenseite losfahren kann

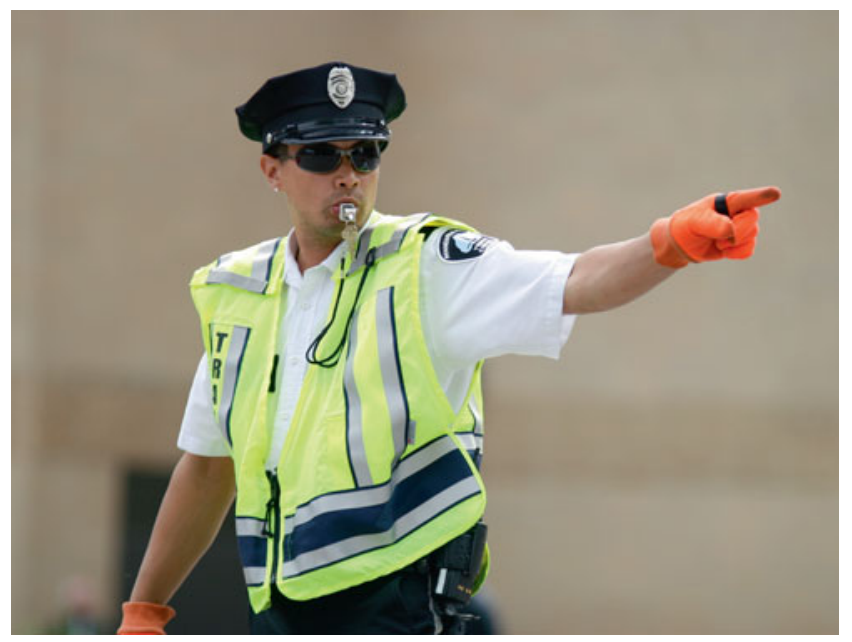


Abb. 7.7 Ein Polizist in Bankok winkt dem Fahrzeug zum Abbiegen

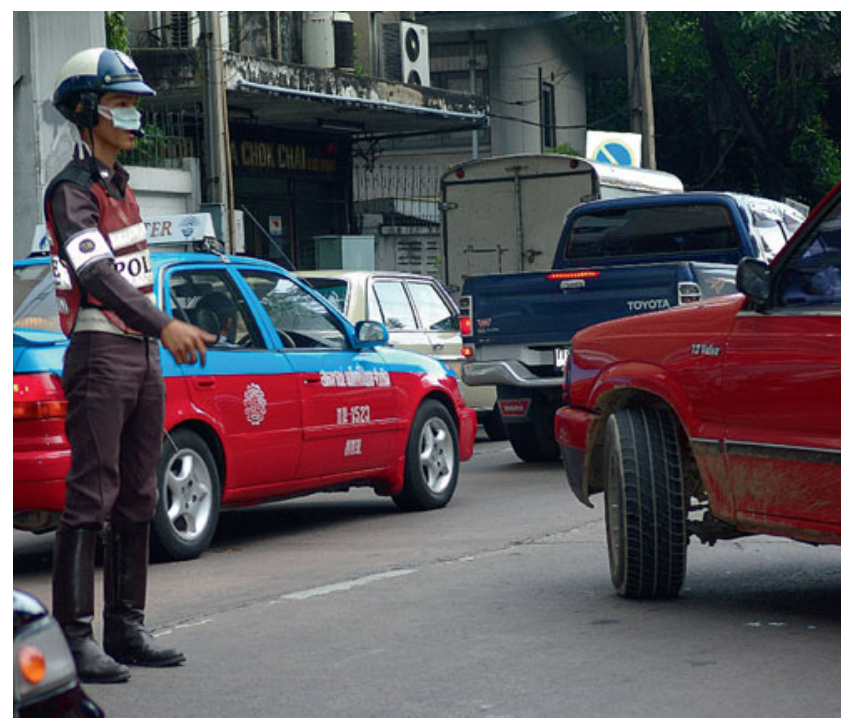

Abb. 7.8 Ein Polizist in Schweden fordert das Fahrzeug zum Nachrücken auf

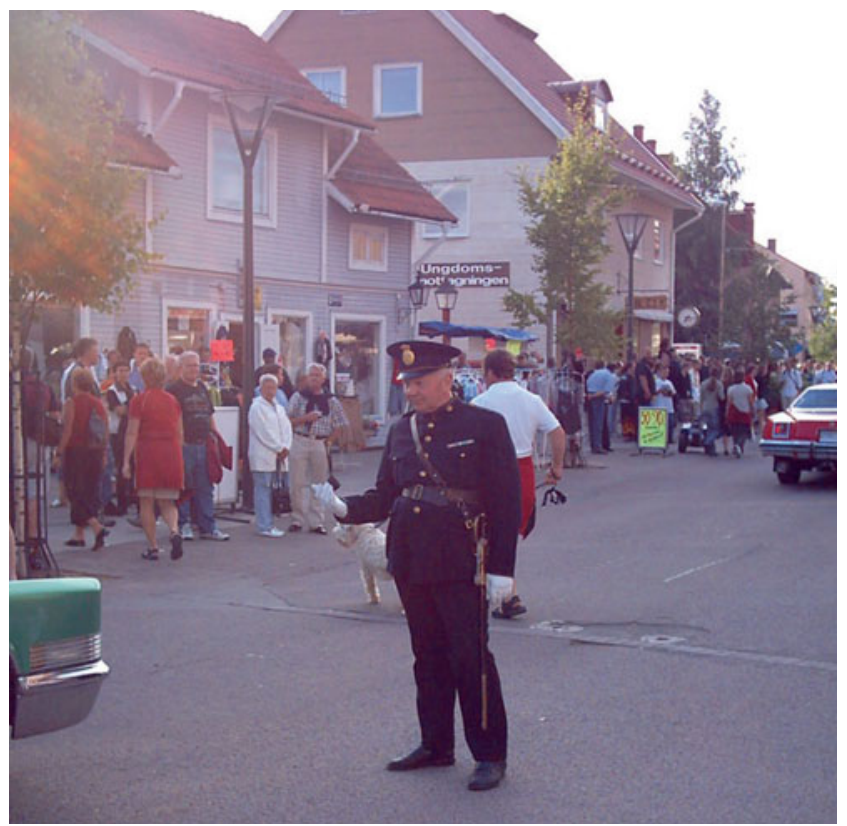




\subsection{Auswirkungen der Kommunikationsmöglichkeit auf die Akzeptanz autonomer Fahrzeuge}

Unter dem Gesichtspunkt der Akzeptanz muss man sich nochmal den Effekt der informellen Kommunikation in „Verhandlungssituationen“ vergegenwärtigen. Diese dient unter Berücksichtigung von $\S 1$ der STVO zur Auflösung von Situationen, die zwar prinzipiell geregelt sind, bei denen die Befolgung der Regel aber zu einer erheblichen Störung des Verkehrs führen würde.

\section{Beispiel 1}

Wegen eines parkenden oder liegen gebliebenen Fahrzeugs in der eigenen Fahrspur ist es erforderlich, auf die Gegenfahrbahn zu fahren und dabei im Extremfall eine durchgezogene Linie zu überfahren. Bei dichtem Gegenverkehr erfordert das eine Abstimmung mit dem Gegenverkehr. Abgesehen von dem (verbotenen) Überfahren der durchgezogenen Linie kann die Situation ohne ,Verhandlung“ gelöst werden, wenn der Gegenverkehr so stark abnimmt, dass die Benutzung des anderen Fahrstreifens auch ohne Verständigung zwischen den Fahrzeugen möglich ist. Mit zunehmender Verkehrsdichte wird eine Verhandlung mehr und mehr erforderlich.

Wie signalisiert der Gegenverkehr seine Kooperationsbereitschaft? Er muss zum einen verzögern, um eine prinzipielle Möglichkeit zum Ausscheren auf die Gegenfahrbahn zu eröffnen. Verzögern allein reicht meist als Signal nicht aus, denn es kann viele Gründe geben, warum ein Fahrzeug eine etwas größere Lücke zum Vorausfahrenden herstellt. Deshalb zeigt der Gegenverkehr in der Regel seine Absicht durch Verzögern und zusätzlich durch die Betätigung der Lichthupe an. Erkennt nun das autonome Fahrzeug dieses Signal nicht, so wird es als „Verkehrshindernis“ betrachtet, was für die Akzeptanz sicher nicht günstig ist.

\section{Beispiel 2}

Autonome Fahrzeuge werden sich, wie eben gezeigt, primär vorsichtiger verhalten als menschliche Autofahrer, da sie wenig Wissen über den Kontext und informelle Zeichen besitzen. Sie können aber auch Manöver fahren, die von einem menschlichen Fahrer normalerweise nicht ausgeführt werden. Im Gegensatz zu menschlichen Autofahrern reagieren sie schneller, d.h., die als ,Schrecksekunde“ bezeichnete Reaktionszeit des Menschen entfällt weitestgehend. Dies ist einer der Gründe, warum autonome Fahrzeuge weniger Unfälle verursachen würden. Weiterhin ist aus der Unfallforschung bekannt, dass viele Fahrer das Verzögerungspotenzial ihrer Fahrzeuge nicht voll ausschöpfen und auch beim Ausweichen die physikalischen Grenzen entlang des Kamm'schen Kreises im Allgemeinen nicht erreichen. Fahrer-Assistenz-Systeme wie automatische Notbremse oder Ausweichassistent wollen diese Defizite kompensieren. In Notsituationen könnte also ein autonomes Fahrzeug nicht nur schneller, sondern auch an den physikalischen Grenzen der Längs- und Querbeschleunigung agieren. Wie sich das auf andere Verkehrsteilnehmer auswirkt, ist momentan weitgehend unbekannt. Ein einfacher Lösungsansatz wird in Abschn. 7.6 diskutiert. 


\subsection{Mit welchem mentalen Modell werden andere Verkehrsteilnehmer auf Fahrfehler von autonomen Fahrzeugen reagieren?}

Nahe verwandt mit der vorher genannten Interaktion und Verhandlung ist die Kompensation von Fehlern anderer Verkehrsteilnehmer. Neben der Vermeidung von unmittelbaren Kollisionen durch Ausweichtrajektorien gehört die Toleranz der Fahrfehler anderer zum alltäglichen Straßenverkehr. Zunächst muss geklärt werden, was unter „Fahrfehlern“ zu verstehen ist. Fahrfehler können aus Sicht eines menschlichen Fahrers streng regelkonformes, aber situationsangepasst nicht optimales Verhalten darstellen.

So kann beispielsweise ein Fahrzeug mit einem menschlichen Fahrer, das sich auf einer bevorrechtigten Straße befindet, das autonome Fahrzeug aus der untergeordneten Straße zunächst passieren lassen, weil es sonst nicht in die untergeordnete Straße einbiegen könnte. Der menschliche Fahrer würde also auf sein regelkonformes Vorfahrtsrecht verzichten, was vom autonomen Fahrzeug sicher erkannt werden müsste.

Fahrfehler können aber auch Defizite im Verhaltensrepertoire des autonomen Fahrzeugs zur Lösung spezieller Situationen oder das Erreichen von Systemgrenzen sein.

\section{Beispiel}

Ein autonomes Fahrzeug geht im Modus „Staupilot“ in einen sicheren Zustand, weil die Systemgrenzen erreicht sind. Das bedeutet: Das Fahrzeug bremst in den Stillstand. Obwohl prinzipiell das Bremsen bis in den Stillstand im Stau häufig vorkommt, werden die übrigen Verkehrsteilnehmer zumindest verwirrt sein, wenn alle anderen Fahrzeuge fahren und nur das autonome stehen bleibt. Erfolgt das Manöver relativ abrupt und (für andere) aus nicht ersichtlichem Grund, so kann daraus eine Gefährdung entstehen. Allerdings sollte man berücksichtigen, dass sich im aktuellen Straßenverkehr Verkehrsteilnehmer auch nicht fehlerlos verhalten und ihre Fehler meist durch andere kompensiert werden. Somit stellt sich die Frage: Welche Eigenschaften weisen Menschen den autonomen Fahrzeugen zu. Werden sie eher als weniger kompetent im Vergleich zu menschlichen Fahrern eingeschätzt, oder gelten sie als perfekt funktionierende Automaten?

Ziel einer Einführungsstrategie muss die Prägung eines positiven und zugleich realistischen Bildes autonomer Fahrzeuge im Bewusstsein aller Verkehrsteilnehmer sein. Dann, und nur dann werden autonome Fahrzeuge einen adäquaten Platz im System Verkehr einnehmen. Die Voraussetzungen hierfür sind prinzipiell gegeben. So ist aus Umfragen zu neuen Technologien wie etwa der Robotik bekannt, dass Europäer eine positive Einstellung gegenüber Robotern aufweisen [12]. Auch Fahrerassistenzsysteme, als Vorstufe zu hochautomatisierten und schließlich autonomen Fahrzeugen, erfreuen sich mittlerweile eines hohen Ansehens als nützliche Helfer und werden zunehmend von den Käufern nachgefragt [13].

Neben der Einstellung gegenüber technischen Systemen ist die Zuweisung von Fähigkeiten und Eigenschaften neuer technischer Systeme vom Wissensstand der Nutzer abhängig. Naive Verhaltensmodelle von technisch nicht versierten Personen weisen technischen 
Systemen in der Regel mehr Fähigkeiten zu, als sie wirklich besitzen. Die aktuelle, vor allem marketingorientierte Demonstration von autonomen Fahrzeugen in den Medien vermittelt den Eindruck, dass diese Fahrzeuge alle Situationen beherrschen können. Damit werden die Erwartungen an autonome Fahrzeuge so hoch, dass Fahrfehler zumindest zur Irritation, wenn nicht zu Sicherheitsproblemen führen. Um ein realistisches Verständnis für mögliche Probleme zu erzeugen, ist es daher essenziell, frühzeitig zu vermitteln, wo die Fähigkeiten und die Grenzen autonomer Fahrzeuge liegen.

\subsection{Kulturelle Unterschiede}

Im Zusammenhang mit kulturellen Unterschieden stellen sich verschiedene Fragen: Gibt es universelle Grundregeln für nonverbales Verhalten, die nur adaptiert werden müssen? Wie lassen sich kulturelle Unterschiede in der Kommunikation und der Erwartungshaltung auf das Kommunikations- und Entscheidungsverhalten eines Fahrroboters übertragen? Wenn ja, wie lassen sich diese adaptieren? Welche Verhaltensweisen gibt es im internationalen Vergleich?

Die bekanntesten kulturvergleichenden sieben Studien zu nonverbalen Äußerungen anhand des Gesichtsausdrucks stammen von Paul Ekman [14]. Er fand kulturübergreifend einheitliche Gesichtsausdrücke für die Basisemotionen Angst, Ekel, Freude, Trauer, Überraschung, Wut und Verachtung.

Die meisten dieser Basisemotionen treten zwar auch im Straßenverkehr auf, die Bedeutung für die Kommunikation mit anderen ist aber nur von eingeschränkter Wichtigkeit. Eine besondere Bedeutung hat der Ja/Nein-Code in Verhandlungssituationen. In Mittelund Nordeuropa und den USA gilt Kopfnicken als Bejahung und Kopfschütteln als Verneinung. Im Gegensatz dazu wird in Indien, Pakistan, aber auch in Bulgarien das Kopfwackeln von Schulter zu Schulter, das dem „Nein“ in Europa und USA ähnelt, für „Ja“ verwendet. Schließlich gibt es noch eine Art, Ja und Nein nonverbal anzuzeigen, die in Griechenland, der Türkei und Süditalien verbreitet ist. Das „Ja“ wird durch eine Fallbewegung des Kopfes nach vorne ausgedrückt, für ein „Nein“ wird der Kopf in den Nacken geworfen ([15], S. 134). Missverständlich für die Interaktion zwischen Verkehrsteilnehmern kann auch noch das Heranwinken mit einer Handgeste sein. Die sogenannte „Paddelgeste“ [15] mit nach unten gerichteter Handfläche dient in Japan und im Mittelmeerraum zum Heranwinken. In England und Deutschland wird sie eher in der Bedeutung „Geh weg!“ eingesetzt. Trotz kultureller Unterschiede nonverbaler Zeichen, die mit den Händen ausgeführt werden, sind interessanterweise speziell diejenigen Zeichen, die zur Beschimpfung anderer im Straßenverkehr dienen, international weitgehend einheitlich. Der erhobene Zeigefinger (s. Abb. 7.9), das Tippen an die Stirn (s. Abb. 7.10) oder das Vorbeiwischen an der Stirn (s. Abb. 7.11) zum Ausdruck von Unverständnis für das Verhalten zeigen dem anderen an, was man von ihm hält bzw. nicht hält. Einzig der vertikale Kreis aus Zeigefinger und Daumen mit abgespreizten restlichen Fingern bedeutet in Deutschland (meist) „o.k.“ oder Lob, während es in Italien „Arschloch“ bedeutet ( s. Abb. 7.12). 
Abb. 7.9 Erhobener Finger

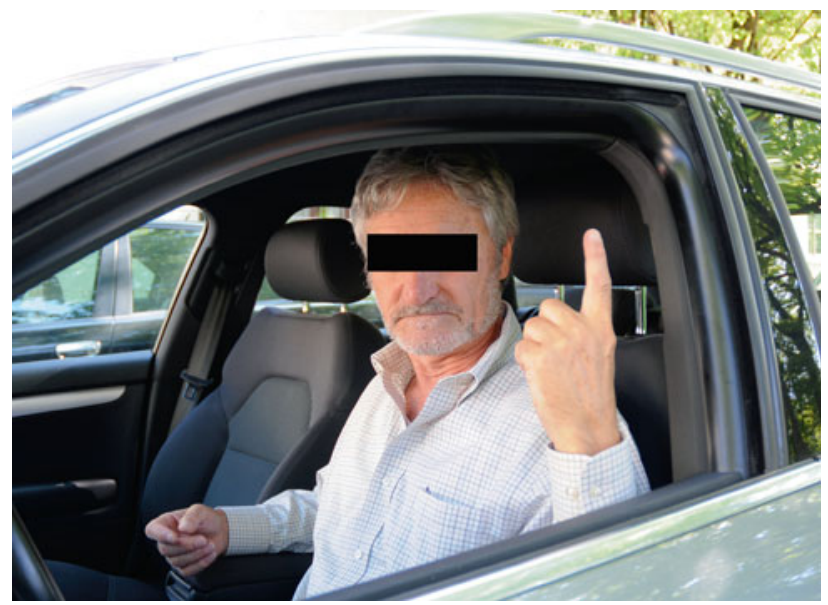

Abb. 7.10 Verärgerung ausdrücken

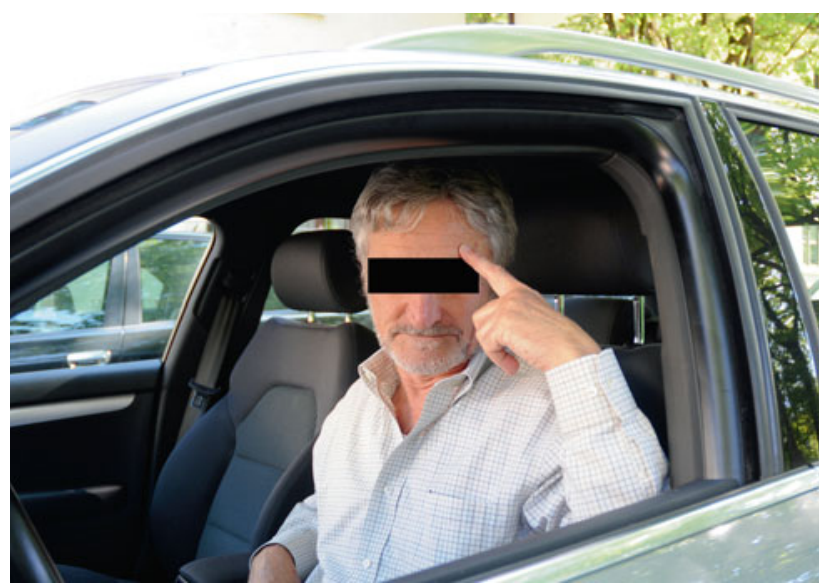

Abb. 7.11 Vorbeiwischen an der Stirn zum Ausdruck von Unverständnis mit dem Verhalten des anderen

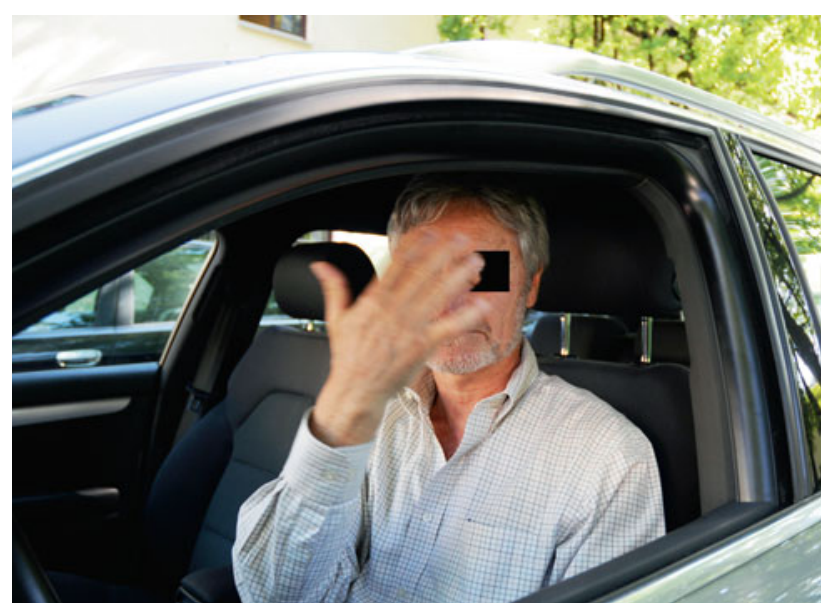


Abb. 7.12 Alles gut!

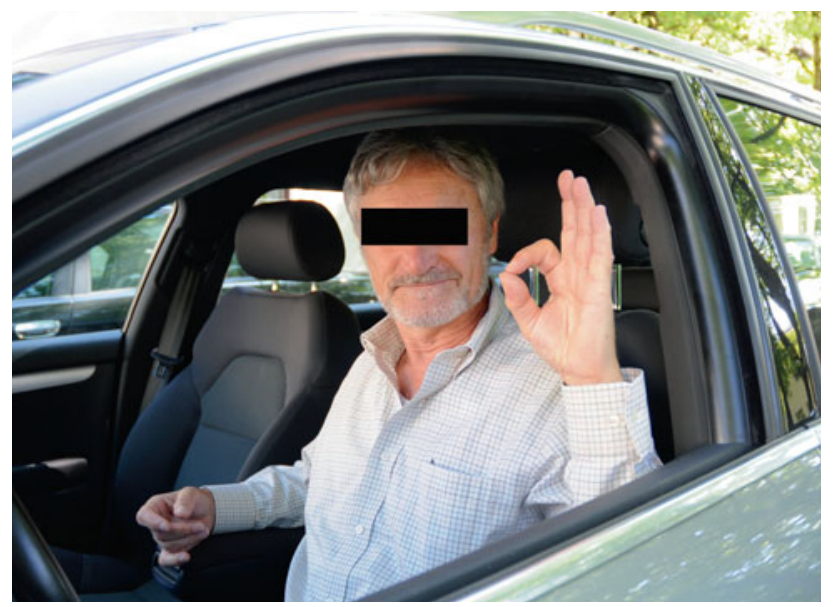

Im Gegensatz zu den kulturübergreifenden Basisemotionen „Ärgerzeichen“ existieren für die informelle Kommunikation zwischen Verkehrsteilnehmern sicherlich kulturelle Unterschiede, die sich im Fahrverhalten ausdrücken und auch einem Wandel unterworfen sind.

Nach allgemeiner Ansicht fahren Südeuropäer dynamischer und zugleich defensiver als Mitteleuropäer. Als informelle Signale zwischen den Fahrzeugen dienen in Südeuropa vor allem die Beschleunigung und die Hupe. Ein Fahrer, der mit einer starken Beschleunigung (eventuell unter Verwendung der Hupe) in eine Lücke einschert, erwartet, dass die anderen Verkehrsteilnehmer nachgeben. Er erwartet aber auch keine weitere Rückmeldung, sondern geht davon aus, dass seine Absicht erkannt und akzeptiert wird.

In Mittel- und Nordeuropa wird der Fahrer des einscherenden Fahrzeugs eher eine Rückmeldung erwarten, zumindest in Form von Blickkontakt oder als Nicken oder Handzeichen. Umgekehrt ist - zumindest in Deutschland - das Beharren auf dem Vorfahrtsrecht verbreitet, sodass ein Einschermanöver ohne das Einholen des „Einverständnisses“ eines anderen Verkehrsteilnehmers selten ist, da ansonsten die Gefahr einer Kollision droht.

Der Verkehr in USA ist durch gleichmäßiges und spurbezogenes Fahren gekennzeichnet. Hier spielen, abgesehen von den offiziellen Zeichen, informelle Zeichen eine geringere Rolle.

In China ist der Verkehr - zumindest bislang - durch eine geringe Regelbefolgung und eine für Ausländer schwer zu durchschauende Kommunikation geprägt. Chinesische Autofahrer sind Meister der Überraschung, ignorieren Verkehrsregeln, Hupen gilt nur als „freundlicher Gruß“ [16].

Von besonderer Bedeutung ist die Kommunikation zwischen Autos und Fußgängern. Die Verständigung erfolgt hier durch Handzeichen, Betreten des Überwegs oder aber auch Warten am Zebrastreifen, bis die Autos anhalten. Welche Auswirkungen hat das auf das Verhalten von autonomen Fahrzeugen? Zum einen werden autonome Fahrzeuge aufgrund der Trajektorie und Beschleunigung des Fußgängers vorhersagen, ob eine Kreuzungs- 
absicht besteht oder nicht. Darüber hinaus gibt es andere Verhaltensweisen, die weniger eindeutig sind: Stehen am Überweg ohne Kreuzungsabsicht (z. B. weil man sich unterhalten will) oder zögerliches Verhalten, das keine eindeutige Querungsabsicht erkennen lässt. In all diesen Fällen würde das autonome Fahrzeug aus Sicherheitsgründen anhalten. Die Konsequenzen sind einerseits ,unberechtigtes Anhalten“ bzw. die Gefahr, dass beispielsweise Kinder oder Jugendliche einen neuen Sport entwickeln, Fahrzeuge am Zebrastreifen zum Anhalten zu zwingen.

Allerdings sind die gesetzlichen Regelungen und auch die tatsächlichen Verhaltensweisen zwischen Ländern sehr unterschiedlich. Während beispielsweise in Italien bis vor einigen Jahren kein Anhaltegebot für Autos am Zebrastreifen galt, ist es jetzt eingeführt worden. Nach Berichten von China-Reisenden ist es nur empfehlenswert, den Zebrastreifen als Fußgängerpulk zu überqueren, weil man als Einzelner keine Chance hat.

Fußgänger, die sich mit Handzeichen verständigen, gehen davon aus, dass die Handzeichen gesehen werden - sie werden aber in der Regel ohne Beachtung der Verzögerung des Verkehrs die Straße nicht überqueren.

\subsection{Kompensationsmöglichkeiten}

Die mangelnden Fähigkeiten von Fahrrobotern zur informellen Kommunikation in „Verhandlungssituationen" ließen sich im ersten Schritt auf einfache Weise lösen.

Durch die eindeutige und sichtbare Kennzeichnung autonomer Fahrzeuge könnte den übrigen Verkehrsteilnehmern die Besonderheit der Fahrzeuge und ihr abweichendes Verhalten verdeutlicht werden. Dies würde anderen Verkehrsteilnehmern zeigen, dass sie nicht das gewohnte Verhalten erwarten können, und in der Folge auch die Akzeptanz in den oben beschriebenen Beispiel-Situationen erhöhen. Auch Fahrschulfahrzeuge sind ja besonders kenntlich gemacht, um andere Verkehrsteilnehmer zu informieren und um Verständnis für ein entweder sehr regelkonformes oder aber unsicheres Verhalten zu bitten.

Für die Kennzeichnung spricht speziell in der Einführungsphase einiges. Wenn - wie in dem Szenario „Valet-Parken“ (s. Kap. 2) vorgesehen - Fahrzeuge ohne Person auf dem Fahrersitz fahren, so kann dies bei den übrigen Verkehrsteilnehmern zu Irritationen führen. „Bewegt sich das Fahrzeug autonom, oder ist es unkontrolliert unterwegs?" Durch die Kennzeichnung stellt sich diese Frage nicht. Die Kennzeichnung von autonomen Fahrzeugen kann zudem einen Marketingeffekt haben, der eine schnelle Verbreitung zur Folge hat. So gab es beispielsweise bei der Einführung von ABS Aufkleber für die Heckscheibe mit dem Hinweis „Dieses Fahrzeug hat ABS“, um anzuzeigen, dass der Bremsweg kürzer ist. Technisch gesehen ist das zwar nicht korrekt, da ABS vor allem die Lenkfähigkeit beim Bremsen erhält, aber unter Marketinggesichtspunkten war der Aufkleber ein Erfolg.

Es spricht aber auch einiges gegen die spezielle Kennzeichnung von autonomen Fahrzeugen. Da sie sich, u. a. wegen ihrer eingeschränkten Verhandlungsfähigkeit, absolut regelkonform verhalten müssen, können sie auch Ziel von unerwünschten Eingriffen von außen sein. 


\section{Ein einfaches Beispiel}

Ein Fußgänger würde eine Straße nicht überqueren, wenn sich ein Auto nähert, da er nicht sicher ist, ob er vom Fahrer gesehen wird und ob der Fahrer bremst bzw. anhält. Bei einem autonomen Fahrzeug kann sich der Fußgänger darauf verlassen, dass das Fahrzeug (unter Berücksichtigung der physikalischen Grenzen) in jedem Fall anhalten wird. Das Stoppen autonomer Fahrzeuge könnte also durchaus ein Sport für Jugendliche werden bzw. Erwachsene dazu verleiten, ohne Rücksicht auf den fließenden Verkehr, die Straße zu überqueren. „Der muss ja anhalten - so ist er programmiert“. Beides ist für den Verkehrsfluss nicht günstig und wird die Akzeptanz autonomer Fahrzeuge nicht positiv beeinflussen.

$\mathrm{Ob}$ der Negativeffekt eintritt, ist schwer vorherzusagen. Es hängt sicher stark von der Art und Weise der Einführung autonomer Fahrzeuge ab. Gelten sie als positive technische Neuerung, der man gewisse Schwächen verzeiht, so dürfte der Negativeffekt kaum auftreten. Gelten sie hingegen als Statussymbol für Privilegierte, so wird der „Neidfaktor“ überwiegen und Versuche, das System zu stören, werden gehäuft auftreten.

\subsection{Neue Kommunikationsformen für einen effektiven Informa- tionsaustausch aus psychologischer und technischer Sicht}

Prinzipiell muss ein autonomes Fahrzeug Gesten bzw. Trajektorien anderer Verkehrsteilnehmer erkennen und interpretieren können. Zur Interpretation benötigt es Situationswissen, um die Zeichen richtig zu deuten. Für die Informationsübermittlung zu anderen Fahrzeugen oder Verkehrsteilnehmern dürften für die meisten Situationen die „offiziellen“ Signale wie Blinker, Hupe und Lichthupe ausreichend sein.

Ein interessanter Ansatz zur Kommunikation zwischen autonomen Fahrzeugen und Fußgängern stammt von der Forschungsgruppe um Kent Larsen vom MIT [17]. In einem Prototyp mit Multisensorik, der allerdings nur entfernt einem realen Fahrzeug ähnelt, wurden mehrere Aktoren eingebaut. Schwenkbare und blinkende LEDs, die wie ein Auge aussehen, wenden sich dem Fußgänger zu und signalisieren ihm: „Ich habe dich gesehen.“

Zusätzlich schwenken gerichtete Lautsprecher zum Fußgänger und sagen ihm, dass er die Straße überqueren kann.

Werden Fußgänger entdeckt, so können LEDs in den Rädern, die die Farbe von Grün zu Orange und Rot ändern, den Fußgängern anzeigen, dass sie erkannt wurden, um sie zu warnen (s. Abb. 7.13).

Ein weiteres Signal, das von autonomen Fahrzeugen an andere ausgesendet werden kann, besteht im „,deutlichen Fahren“.

Will beispielsweise ein autonomes Fahrzeug mit einem anderen kooperieren (z. B. Öffnen einer Lücke zum Einscheren), so müsste es deutlich verzögern, um die Lücke erkennbar zu machen und dem anderen Fahrer die Sicherheit zu vermitteln, dass ein gefahrloses Einscheren möglich ist.

Kommunikation und informelle Regeln unterliegen einem permanenten Wandel. Auf der verbalen Ebene lässt sich das eindrücklich an dem Wort ,geil“" nachvollziehen. Spätes- 


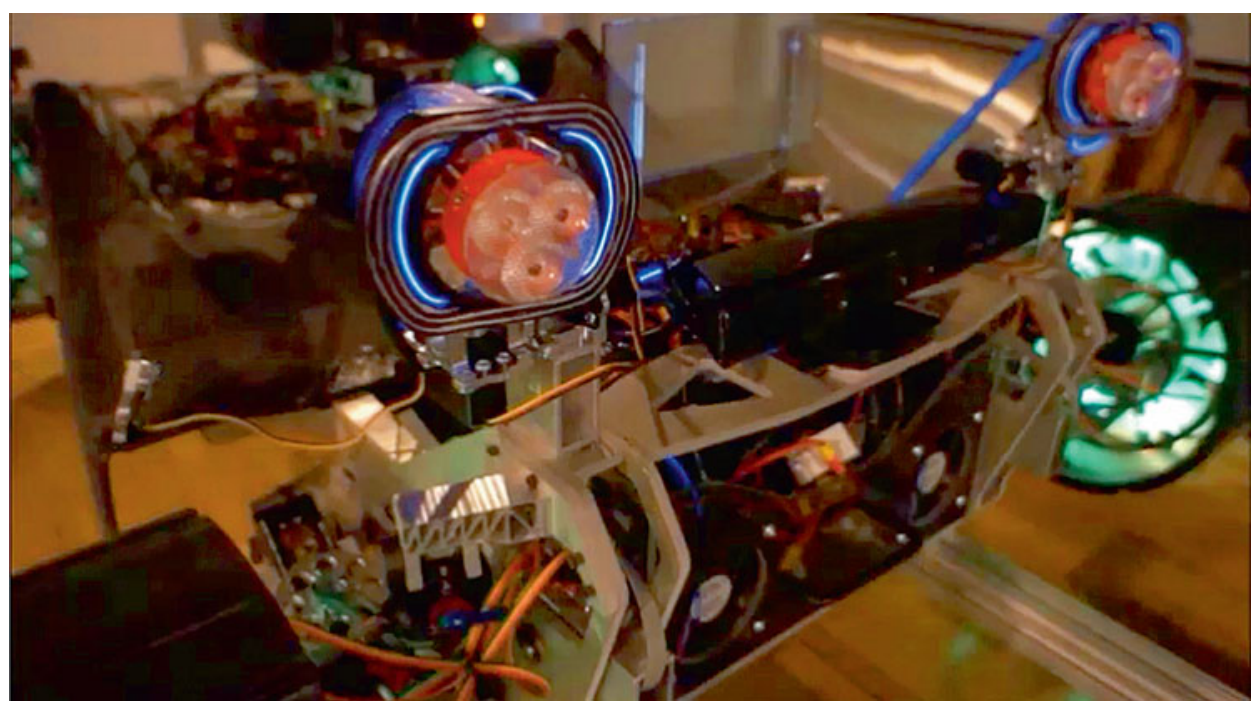

Abb. 7.13 AEVITA: Autonomous Electric Vehicle Interaction Testing Array

tens seit „Geiz ist geil“" hat dieser Begriff aus der Jugendsprache eine völlig andere Bedeutung bekommen. Auch das nonverbale Signal wie der nach oben gestreckte Daumen für „gefällt mir“ ist über die neuen Medien zum aktuellen Standard geworden. Das bedeutet zum einen, dass autonome Fahrzeuge immer wieder neue Zeichen nonverbalen Verhaltens lernen müssten, d.h., ein regelmäßiges Update des Zeichenvorrats und dessen Bedeutung ist erforderlich. Es ist aber auch ein anderer Effekt zu erwarten: Durch die Zunahme autonomer Fahrzeuge mit einem anderen, vor allem an formalen Regeln orientierten Verhalten am Straßenverkehr dürften auch die übrigen Verkehrsteilnehmer dieses Verhalten übernehmen. Damit wird der Verkehr stärker normiert, aber deshalb nicht unbedingt flüssiger ablaufen. Flexible Kooperation zwischen Verkehrsteilnehmern bekommt vor allem bei dichtem Verkehr eine steigende Bedeutung. Die Abstände werden geringer, die Dynamik erhöht sich und die Beachtung von informellen Regeln steigt an.

Eine weitere technische Lösung könnte Car2Car-Kommunikation darstellen. Sie setzt voraus, dass die betreffenden Verkehrsteilnehmer - also nicht nur die autonomen Fahrzeuge - mit der entsprechenden Technik ausgerüstet sind. Car2Car-Kommunikation wird schon lange beforscht, und es existiert eine Reihe von Demonstrationen, auch in größeren Testfeldern, wie etwa simTD [18]. In der Verbreitung von Car2Car (V2V) oder Car-to-X/ V2X (Car to Infrastructure/Vehicle to Infrastructure) könnte ein wesentlicher Lösungsansatz für die Kommunikationsprobleme zwischen autonomen Fahrzeugen und menschlichen Fahrern bestehen.

Frost und Sullivan [19] gehen davon aus, dass bis zum Jahr 203040 Prozent der Fahrzeuge mit V2V-bzw. V2X-Technologie ausgestattet sind, da sich die Nutzer davon große Vorteile wie weniger Staus und eine höhere Sicherheit versprechen. Somit könnte die 
zügige Verbreitung dieser Technik, die zunächst etwas anderes im Fokus hat, Kommunikationsprobleme zwischen autonomen und von Menschen gesteuerten Fahrzeugen mildern.

Jedoch werden auch dann noch unauflösbare Situationen für autonome Fahrzeuge entstehen, entweder, weil die anderen Verkehrsteilnehmer nicht über die entsprechende Kommunikationseinrichtung verfügen, oder die Situation durch einfache Kommunikation nicht aufzulösen ist. Fährt das autonome Fahrzeug ohne Fahrer, der in diesen Fällen die Fahraufgabe kurzzeitig (aber nicht kurzfristig) übernehmen kann, so muss eine übergeordnete Leitstelle eingreifen.

\subsection{Fazit}

Sind die Fahrzeug-zu-Fahrzeug-Kommunikationsmöglichkeiten heute noch sehr beschränkt und wenig verlässlich, so wird gerade mit ungeschützten Verkehrsteilnehmern die Kommunikation mit Blicken, mit Aktionen und Aktionsreihenfolgen vielfältig genutzt. Je nach mentaler Situation wird mehr oder weniger intensiv miteinander verhandelt, z. B. beim Überschreiten des Fußgängerüberwegs. Die dabei angewandten Regeln sind stark kulturell geprägt, insbesondere die Erwartungshaltung an das Gegenüber wird in verschiedenen Kulturen unterschiedlich gesehen, sodass sich keine allgemein gültigen Regeln für den Fahrroboter ableiten lassen. Ein weiteres Problem entsteht allein aus der Nichterkennbarkeit des aktiven Fahrzeugführers, des Roboters. Dies ist zumindest bei fahrerlosen Fahrzeugen offensichtlich. Ist der Fahrerplatz des autonom fahrenden Fahrzeugs aber besetzt, so wird die Kommunikation voraussichtlich falsch adressiert. Ohne Blick- und Gestenerkennung von anderen Verkehrsteilnehmern wird ein Mischverkehr schwierig. Dies gilt vor allem für den Niedergeschwindigkeitsbereich. Mit zunehmenden Geschwindigkeiten nimmt die Bedeutung dieser Kommunikation aus verschiedenen Gründen ab, denn

- sie ist nicht ein-eindeutig,

- sie verlangt vom Partner eine Rückmeldung und dauert daher bei höheren Geschwindigkeiten zu lange,

- die Erfassung durch den anderen Verkehrsteilnehmer ist bei höheren Geschwindigkeiten eingeschränkt, z. B. ist das Blickverhalten in der Kürze der Zeit nicht gut zu erfassen.

Welche Anforderungen ergeben sich daraus für die Absicherung der auf Kommunikation mit anderen Verkehrsteilnehmern basierenden Funktionalität?

Autonome Fahrzeuge müssen sich somit zunächst so verhalten, als gäbe es keine informelle Kommunikation, also komplett regelkonform. Im Falle einer unauflösbaren Situation muss die Fahraufgabe an einen menschlichen Fahrer übergeben werden. Ist kein menschlicher Fahrer vorhanden, so muss eine Leitzentrale in das autonome Fahrzeug eingreifen. Das Verkehrsmanagement würde analog zum Luftverkehr gestaltet, bei dem eine zentrale Leitstelle alle Flug- und Rollbewegungen steuert. An die Stelle des Piloten, 
der Befehle der Leitstelle ausführt, tritt dann der Fahrroboter, der Befehle auf der Manöverebene erhält und diese Aktionen selbstständig auf der Stabilisierungsebene ausführt. Ungeklärt ist dabei noch, wie ein autonomes Fahrzeug erkennen soll, dass die Situation unauflösbar ist und die Leitstelle verständigt werden muss.

\section{Literatur}

1. archive.darpa.mil/grandchallenge/ zuletzt besucht am 25.7.2014

2. Ziegler, J., Bender, P., Lategahn, H., Schreiber, M., Strauß, T., Stiller, C. (2014). Kartengestütztes automatisiertes Fahren auf der Berta-Benz-Route von Mannheim nach Pforzheim. FAS 2014, Uni-DAS, S. 97-94

3. Saust, F., Wille, J.M., Lichte, B., Maurer, M. (2011). Autonomous Vehicle Guidance on Braunschweig's inner ring road within the Stadtpilot Project. In: Intelligent Vehicles Symposium (IV), Baden-Baden. S. 169-174

4. Merten, K. (1977). Kommunikationsprozesse im Straßenverkehr. In: Bundesanstalt für Straßenwesen (Hrsg) Symposion 77. Köln: Bundesanstalt für Straßenwesen

5. Darwin, C. (1874). Der Ausdruck der Gemüthsbewegungen beim Menschen und den Thieren. Halle

6. Pfungst, O. (1907). Das Pferd des Herrn von Osten (Der kluge Hans). Leipzig

7. Schmidt, S., Färber, B. (2009). Pedestrians at the kerb - Recognising the action intentions of humans. Transportation Research Part F: Traffic Psychology and Behavior, 12, S. 300-310

8. Risser, R. (1988). Kommunikation und Kultur des Straßenverkehrs. Wien

9. Heidegger, M. (1927). Sein und Zeit. Tübingen. 11. Auflage 1967

10. Frerichs, K. (1995). Der Winker und das Winken: Ein zeichenphänomenologischer Passus in Martin Heideggers Sein und Zeit. Zeitschrift für Semiotik, Band 17, Heft 1-2. S. $133-142$

11. Savigny, E.v. (1995). Autofahrerzeichen: Funktion, Systeme, Autonomie. Zeitschrift für Semiotik, Band 17, Heft 1-2. S. 105-128

12. Special Eurobarometer 382, Public Attitudes Towards Robots, Report Sept. 2012

13. Continental Mobilitätsstudie http://www.pressebox.de/pressemitteilung/continental-reifendeutschland-gmbh-hannover/Deutsche-Autofahrer-wollen-Automatisiertes-Fahren-fuer-dieAutobahn/boxid/647170 zuletzt besucht am 26.8.2014

14. Ekman, P. (1975). Universals and Cultural Differences in Facial Expressions of Emotion. In: J. Cole (Hrsg.): Nebraska Symposium on Motivation 1971. Bd. 19, University of Nebraska Press, Lincoln

15. Broszinsky-Schwabe, E. (2011). Interkulturelle Kommunikation: Missverständnisse - Verständigung. VS Verlag, Wiesbaden

16. http://www.auto-motor-und-sport.de/news/autofahren-in-china-ein-mann-sieht-rot-5957313. html zuletzt besucht am 7.8.2014

17. http://www.technologyreview.com/view/427743/how-do-you-know-an-autonomous-vehicle has-seen-you/ zuletzt besucht am 8.9.2014

18. simTD deliverable D5.5 (2013) http://www.simtd.de/index.dhtml/object.media/deDE/8154/ CS/-/backup_publications/Projektergebnisse/simTD-TP5-Abschlussbericht_Teil_A_Manteldokument_V10.pdf

19. Strategic Analysis of the European Market for V2V and V2I Communication Systems (2014) zitiert nach http://www.frost.com/prod/servlet/press-release.pag?docid=290129681 